\title{
On the meaning of approximate reasoning - An unassuming subsidiary to Lotfi Zadeh's paper dedicated to the memory of Grigore Moisil -
}

\author{
H.-N. L. Teodorescu
}

\author{
Horia-Nicolai L. Teodorescu \\ Gheorghe Asachi Technical University, \\ Iasi, Romania \\ Institute for Computer Science, \\ Romanian Academy, Iasi Branch
}

\begin{abstract}
The concept of "approximate reasoning" is central to Zadeh's contributions in logic. Standard fuzzy logic as we use today is only one potential interpretation of Zadeh's concept. I discuss various meanings for the syntagme "approximate reasoning" as intuitively presented in the paper Zadeh dedicated to the memory of Grigore C. Moisil in 1975.
\end{abstract}

Keywords: logic, truth value, natural language, inference.

\section{Introduction}

I perceive three central ideas in Zadeh's wide conceptual construction in his work until now. The first one is that words and propositions in natural languages, consequently human thoughts are not representable by standard sets and standard binary predicates. The second central idea in many of Zadeh's papers is that humans perform computations in an approximate manner that real numbers can not represent well. The third idea, which Zadeh presented in his more recent works, is that of granularity of human mental representations and reasoning. These three strong points were represented by Zadeh in various forms and synthesized in the title of his paper on "computing with words" [1].

Most frequently - and Zadeh himself is doing no exception - authors cite as the initial paper clearly stating the approximate reasoning model the one published in the journal Information Sciences, July 1975 [2]. However, another paper published in Synthese [3], during the same year, deals with the main ideas in approximate reasoning, not to mention a conference paper published in 1974.

The paper published in Synthese is important in several ways. First, it offers clear explanations of the meanings associated by Zadeh to the syntagme "approximate reasoning". Second, the paper was published in a well-established journal that "spans the topics of Epistemology, Methodology and Philosophy of Science," thus boldly reaching to a large audience in a set of fundamental disciplines and daringly affirming the importance of the new concepts. Third, the paper is important for the Romanian scientists because it re-enforces the understanding of Grigore Moisil's contributions and the deep connections between Zadeh and Moisil. For an in-depth coverage of the last topic, see [4], [5].

Zadeh starts the paper [3] from the common-sense remark that "It is a truism that much of human reasoning is approximate rather than precise in nature." From that remark, he builds a broad program for research.

The program that Zadeh establishes remains, in my opinion, unfinished. In the abstract of the paper discussed here, Zadeh states "Since $T$ [the truth-value set] is not closed under the operations of negation, conjunction, disjunction and implication, the results of an operation on truth-values in $T$ requires, in general, a linguistic approximation by a truth value in $T$." With 
this clarification, classic fuzzy logic as we know today is not a proper representation of Zadeh's original ideas.

There are two ways to interpret the above quotation. The first interpretation is in the frame of the classic thinking and runs as follows. Because language is a set of propositions (we restrict the discussion to truth-valuable propositions only), under whatever logic, all simple and composed propositions have a truth value. Denoting the set of truth values by $T$, the above remark by Zadeh has no effect. Moreover, when defining the truth-valuation function we already need to know the set of truth values, which again makes ineffective Zadeh's remark. Standard fuzzy logic pursue this direction of thinking, as it starts with . Under this approach, as $T$ is given, what is needed is to define the logic operators for the respective logic.

The second interpretation is constructive. It may start with the assumption that language is not predetermined and must be constructed as a recursive, dynamic process, as poets, writers, other language professionals, and laymen do every day. Whenever a new proposition is invented, it is assigned a meaning. The meaning includes what we conveniently name "truth", a coverage degree of the reality that we need for making inferences. The truth may or may not be numerically representable. Moreover, the truth of a composed proposition may not be representable by the truth of each of the initial propositions. Therefore, the "set of truth values" evolves continuously. That, in turn, creates a stumbling block. Because we are supposed to know the truth of the original sentences, but not of the result, how are we supposed to infer? The answer proposed by Zadeh is that we still have an approximate truth in the initial set of truth values, approximating the truth of the new proposition. He hypothesizes that there is always an inverse application, which I will name projection, from the new truth set to the original one, indicating a truth degree in the original set that approximates the truth degree of the composed proposition. That makes our reasoning possible, if approximate.

This way of thinking, more or less directly suggested by Zadeh, opens the door to several formal descriptions. I sketch below a loose formalism for the recursive approach.

Consider a language $L=\left(\{p\}, c_{1}, c_{2}, \cdots c_{r}\right)$, where $p$ denotes extant propositions and $c_{k}$ logic operators (either unary or binary). Notice that $L$ is an initial language, meaning that the initial set $\{p\}$ is evolvable, that is, it is recursively increased by adding propositions correctly formed from the initial ones through logic operations. Assume that any proposition has a characteristic named truth value. Thus, there is some set $T$ of truth values (I use for this set the same notation as Zadeh's), as well as an application such that for any proposition $p$ there is a truth value in $T$. Also assume that any propositions can be concatenated to produce a new or extant proposition. Whenever such proposition is new in the language, it has a characteristic truth value which is not necessarily in $T$. We can regard the creation of new propositions (including those used for reasoning) as producing an application $T \rightarrow T \prime$, as for the negation operator, or $T \times T \times \cdots \rightarrow T$, as for the connectives. The new $T$-set which includes the original one. Moreover, for satisfying Zadeh's hypothesis, there is a projection operation $\vartheta$. Notice that whenever a new proposition is produced, $L$ is modified.

Because of $T$ non-closure to logic operations, we can not talk about a $T$ (proper) set for a language. Instead, we can conceive an object named $T^{*}$-set that can be seen as a string of sets, each generated recursively from the previous $T$-set or from a product of $T$-sets. The string must be complemented by a string of applications from a $T$-set (or from a product of such sets) to the next one in the sequence. Next, we need to allow for a construction where the term approximate makes sense. Several mathematical constructions may be used in this respect, namely the concept of topological space, where approximate may mean "in a vicinity of", the concept of metric space or one of its variants, as pseudo-metric space, where the term approximate may be interpreted as "at a distance less than epsilon", or a measurable space, where the truth may be conceived as elements of the space and the approximation is interpreted as 
On the meaning of approximate reasoning

- An unassuming subsidiary to Lotfi Zadeh's paper dedicated to the memory of Grigore Moisil

"the difference between the measures of the respective two truth values (here, a value can be represented for example by a measurable set) is less than epsilon." The version based on the measurable spaces corresponds to the probabilities. The same version is adopted below, but with no direct connection to probabilities.

The main requirements for the $T^{*}$-set are: i) The truth-attribute of a proposition is represented by an application $\theta: L \rightarrow T$, where $T$ is measurable. ii) For any two extant propositions $p_{1}$ and $p_{2}$ and for any connective $c$, the new proposition $p_{1} c p_{2}$ has a truth degree such that $T \cup\left\{\theta\left(p_{1} c p_{2}\right)\right\}$ is measurable and includes the measurable space $T$. iii) For any valid linguistic construction that uses logic operators, there is an application $\vartheta: T \prime \rightarrow T \prime$, named back-projection. iv) For any connective $c$, there is a formula $f_{c}$ such that $\vartheta\left(\theta\left(p_{1} c p_{2}\right)\right)$ and $f_{c}\left(\theta\left(p_{1}\right), \theta\left(p_{2}\right)\right)$ are two points close enough in $T$.

Standard fuzzy logic obviously satisfies the above conditions, with $T=T$. Replacing the measurable space with a metric one, we obtain another construction, which may be closer to Zadeh's intuition in [3]. Using distances $d: T \rightarrow T$, the conditions to satisfy are:

$$
\begin{gathered}
d(\vartheta(\neg p), 1-\theta(p))<\varepsilon \\
d(\vartheta(p \bigwedge q), \min (\theta(p), \theta(q))<\varepsilon \\
d(\vartheta(p \bigvee q), \max (\theta(p), \theta(q))<\varepsilon,
\end{gathered}
$$

where $\varepsilon$ is the allowed approximation error.

As Grigore Moisil has done, Zadeh emphasizes in all his work how much language is fundamentally creative. By stating that " $T \cdots$ is not closed under the logic operations," he highlights that there is always generation of new meaning, or at least an evolution of the meaning, whenever a sentence is uttered. I believe that this is one of the fundamental contributions made by Zadeh's work until now.

Understanding the creative process in languages and in reasoning was significantly elucidated by Zadeh's work, yet much remains to be done for deriving conclusions and theoretical developments in the directions pointed to by his works. The developments informally suggested above may indicate several such directions, yet others may be put forward in a more formal manner during the years to come.

Acknowledgment. I thank several colleagues and the referees for their comments and suggestions. The note was not supported by, but is related to research performed in the frame of the Romanian Academy.

\section{Bibliography}

[1] L.A. Zadeh, Fuzzy logic = computing with words. Fuzzy Systems, IEEE Transactions on, Volume: 4 Issue 2, pp. 103-111 (May 1996)

[2] L.A. Zadeh, The concept of a linguistic variable and its application to approximate reasoning - I, Information Sciences, vol. 8, no. 3, pp. 199-249, July 1975.

[3] L.A. Zadeh, Fuzzy Logic and Approximate Reasoning (In Memory of Grigore Moisil). Synthese, vol. 30, (1975), pp. 407-428, D. Reidel Publishing Co, Dordrecht, Holland 
[4] G. Georgescu, A. Iorgulescu, S. Rudeanu, Grigore C. Moisil (1906 - 1973) and his School in Algebraic Logic. Int. J. Computers, Communications \& Control, Vol. I (2006), No. 1, pp. 81-99

[5] Z. Lemnaouar and A. Abdelaziz, On the representation of L-M algebra by intuitionistic fuzzy subsets. ARIMA J., Volume 4 (2006), pp. 72-85 